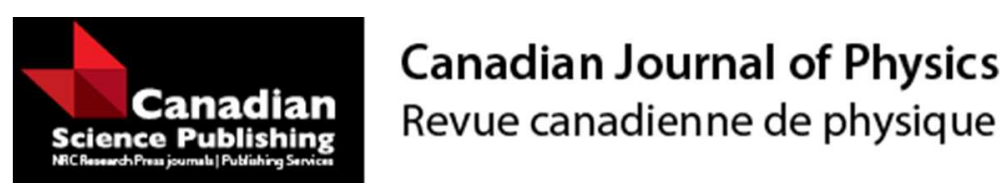

Vibrational spectra and phonon dispersion analysis of a singlewalled zigzag carbon nanotube: A first principles study

\begin{tabular}{|r|l|}
\hline Journal: & Canadian Journal of Physics \\
\hline Manuscript ID & cjp-2016-0421.R1 \\
\hline Manuscript Type: & Article \\
\hline Date Submitted by the Author: & 26-Jul-2016 \\
\hline Complete List of Authors: & $\begin{array}{l}\text { sharma, deepa; National Institute of Technology, } \\
\text { jaggi, neena; National Institute of Technology }\end{array}$ \\
\hline Keyword: & Simulation, molecular modeling, CASTEP, Phonon dispersion, Spectroscopy \\
\hline \multicolumn{2}{|l}{} \\
\hline
\end{tabular}

SCHOLARONE $^{\text {m }}$

Manuscripts 


\title{
Vibrational spectra and phonon dispersion analysis of a singlewalled zigzag carbon nanotube: A first principles study
}

\author{
Deepa Sharma $^{1 *}$, Neena Jaggi ${ }^{2}$ \\ Shaheed Udham Singh Government College, Matak-Majri (Indri), Haryana-India ${ }^{{ }^{*}}$ \\ National Institute of Technology, Kurukshetra-(Haryana)-India ${ }^{1,2}$ \\ Corresponding author: Deepa Sharma (email: bhargava.dp@gmail.com)
}

\begin{abstract}
:
This paper reports a vibrational spectroscopic study on a zigzag singlewalled carbon nanotube (SWCNT) using first principles method based on density functional theory (DFT). The most suitable exchange correlation functional for density functional theory analysis was determined by comparing the predicted value of band gap of the SWCNT under study with the experimental value reported in the literature. General Gradient approximation (GGA) functional in combination with revised-Perdew-Burke-Ernzerh (RPBE) sub-functional was found to give the best results. Using this optimum combination, phonon density of states and phonon dispersion curves have been determined. The analysis of results obtained focuses on symmetry considerations, group theory analysis, segregation of Raman active and IR active vibrational modes and interpretation of the Raman and IR spectra obtained. The earlier approaches on the problem rely upon zone folding technique and force constant models in which structural relaxation factor is not taken care of. An ab initio approach has been adopted by the authors in this work which is advantageous as it neither depends on some predefined parameter nor does it ignore the structural relaxation factor. The analysis of raman spectrum reveals some additional peaks other than the commonly known RBM, D, G \& G' bands in the SWCNT spectra which have been recently reported to be observed experimentally also. Similarly, the theoretically developed IR spectrum for the simulated SWCNT is also in agreement with experimental observations. The methodology presented, thus provides a very useful \& novel simulation route to predict the vibrational modes, Raman spectra and IR spectra of SWCNTs theoretically.
\end{abstract}

Keywords: phonon density of states; phonon dispersion; normal vibrational modes; raman active modes; IR active modes; Simulation; density Functional Theory; molecular modeling; CASTEP; SWCNT

\section{Introduction}

Singlewalled Carbon nanotubes (SWCNTs) have emerged as a wonder material during the last decade. Because of their unique electrical, mechanical and thermodynamic properties, these cylindrical tubes are finding use in a wide variety of applications [1-7].SWCNTs in particular, show fundamental phenomena ranging from possible superconductivity [8] or Luttinger-liquid state [9] to Peierls distortion [10].This paper reports a spectroscopic study on a zigzag singlewalled carbon nanotube. The work presented in this paper develops an insight into the phonon dispersion, normal modes of vibration, Raman spectra and IR spectra of a zigzag singlewalled carbon-nanotube using Cambridge Sequential Total Energy Package (CASTEP) Module of the Material Studio software version 7.0. 
The phonon dispersion curves over the full Brillouin zone are useful in various fields of research like inelastic neutron scattering [11], Second-order Raman spectroscopy [12], double-resonance processes [13-16] and the electron-phonon interactions [17-21]. In most of the studies reported earlier, phonon structure of SWCNTs has been derived from the phonon structure of graphene using zone folding method. However, zone-folding of the graphene phonons does not always give the correct dispersion relation for a SWCNT (especially in the low frequency region) and some additional physical concepts need to be introduced. The calculation of the phonon dispersion has been made using ab initio linear response method. Earlier work on this problem using zone folding and force constant models [22-25] shows that the zone-folding method has two shortcomings: the structural relaxation effect is completely ignored which makes the transferability of the phonon spectra between the graphene and the SWCNT's a serious question and secondly, the vibrational eigenvectors are also different for graphene \& SWCNT. The ab initio approach is advantageous because such calculations do not depend on any predefined parameter. There are different authors that have reported ab initio phonon dispersion of SWCNT's, all using the super cell approach [26-28].The largest differences between ab initio calculations and zone-folding occur in the low frequency range and for tubes with small diameters (less than $0.7 \mathrm{~nm}$ ) also in the high frequency range.

\section{Software and Simulations}

\subsection{Software}

The simulation software used is Materials Studio version 7.0, which is a user friendly and advanced Materials Simulation Software. Materials studio software speeds up the research experiments because one can predict and analyze the results of the experiments and tests. By use of the software, number of experimental trials is reduced; therefore, cost of experiments is also reduced. In the present work, spectroscopic study has been performed on a $(9,0)$ zigzag SWCNT using modeling and simulation.

\subsection{Simulations}

The structural unit was simulated via molecular modelling which comprised of 144 valence electrons in the 36 carbon atoms of the specific nanotube giving rise to 87 energy bands in its electronic band structure. The band gap studies were performed using CASTEP Module. Suitable choice of various tolerance parameters is very crucial for geometry optimization. The most suitable exchange correlation functional was determined. On comparison with the experimental results, the calculation performed using GGA (RPBE) was found to deliver the best match. Normconserving pseudo-potential was used in the reciprocal lattice with fixed basis set quality using Gaussian smearing scheme without any periodic dipole correction. BFGS algorithm which implements variable cell method with geometry line minimiser was initiated to calculate the ground state eigen values, eigen functions and density. Continuing with the ground state wavefunction thus determined and ground state density thus calculated, general kpoint calculations for the density of states, Raman \& IR spectra were performed.

Total 108 phonon modes were calculated at 23 wave-vectors. Group theory was employed to analyse the various phonon modes calculated. Raman active modes and IR active modes were then segregated to extract spectroscopic information out of the calculated data. 


\section{Results and Discussions}

\subsection{Geometry Optimization}

Geometry of the system was optimized w.r.t. energy change, max. displacement, max. force and max. stress. Normconserving pseudo-potential was used in the reciprocal lattice with fixed basis set quality using Gaussian smearing scheme without any periodic dipole correction. Lattice parameters obtained after geometry optimization were $\mathrm{a}=10.39 \mathrm{~A}^{0}, \mathrm{~b}=10.39 \mathrm{~A}^{0} \& \mathrm{c}=4.26 \mathrm{~A}^{0}$ (Cell Volume $\left.=398.5\left(\mathrm{~A}^{0}\right)^{3}\right)$ with cell angles $90^{0}, 90^{0} \& 120^{0}$ confirming the suggested hexagonal geometry (Fig. 1). The unit chosen comprised of 36 units. As expected, the first Brillouin zone obtained was also hexagonal. Supercell approach was adopted. 6 k-points were chosen for BZ sampling. .

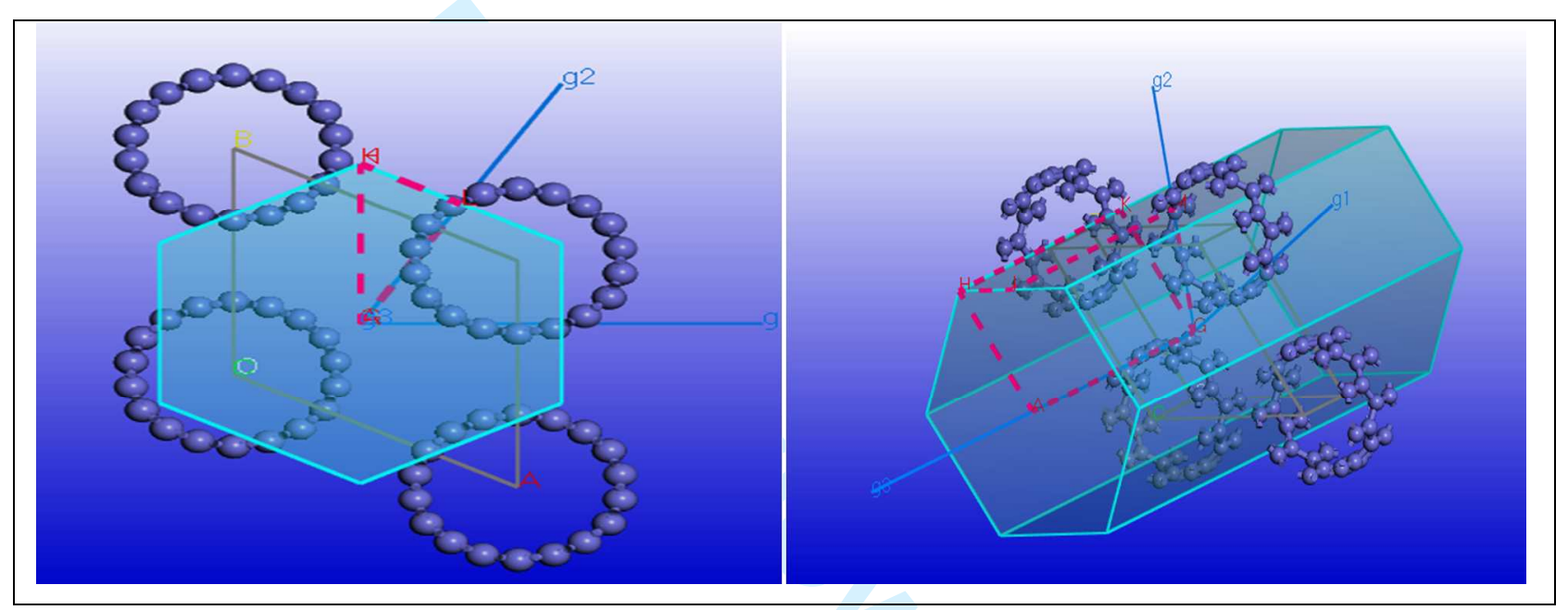

Fig. 1 Lateral and 3-D view of the Optimized geometry obtained by supercell approach

\subsection{Exchange Correlation Optimization}

Band gap study was performed to determine which exchange correlation functional available is the most suitable for further studies to be performed. Following results (Table-1) were obtained

TABLE-1

\begin{tabular}{|c|c|c|c|c|}
\hline \multicolumn{5}{|c|}{ BAND GAP EVALUATION USING CASTEP : (9,0) SINGLEWALLED CARBON NANOTUBE } \\
\hline LDA & \multicolumn{4}{|c|}{ GGA } \\
\hline CA-PZ & RPBE & PBESOL & WC & PW91 \\
\hline $0.190 \mathrm{eV}$ & $\mathbf{0 . 0 9 7 e V}$ & $0.161 \mathrm{Ev}$ & $\mathbf{0 . 0 6 3 e V}$ & $0.003 \mathrm{Ev}$ \\
\hline
\end{tabular}

Comparing these results with the measurements made by Leiber et al. $\left(\mathrm{E}_{\mathrm{g}}=0.080 \mathrm{eV}\right)$ [29], it can be seen that GGARPBE / GGA-WC exchange correlation approximation is the most effective theoretical tool for predicting band gaps in SWCNTs.

\subsection{Phonon Dispersion}

As band gap studies revealed GGA-RPBE exchange correlation approximation to be the most effective, the same was used for further phonon dispersion and spectroscopic studies. Pseudo atomic calculations performed on 
$\mathrm{C}\left(2 \mathrm{~s}^{2} 2 \mathrm{p}^{2}\right)$ Converged in 17 iterations to a total energy of $-145.6 \mathrm{eV}$. Pulay density mixing treatment with Gaussian smearing was used with finite basis set correction. It should be pointed out that different space groups having the same Bravais lattice can have different $\mathrm{K}$ sets in the BZ. For the hexagonal lattice, the K set in the holohedric space groups (with the highest point symmetry D6h) consists of G, A, K, H, M, and L points, which are isolated symmetry points. [30]. Phonon density of states and phonon dispersion curves determined using linear response phonon calculation with the phonon sum rule are shown here (Fig. 2(a \& b) ).

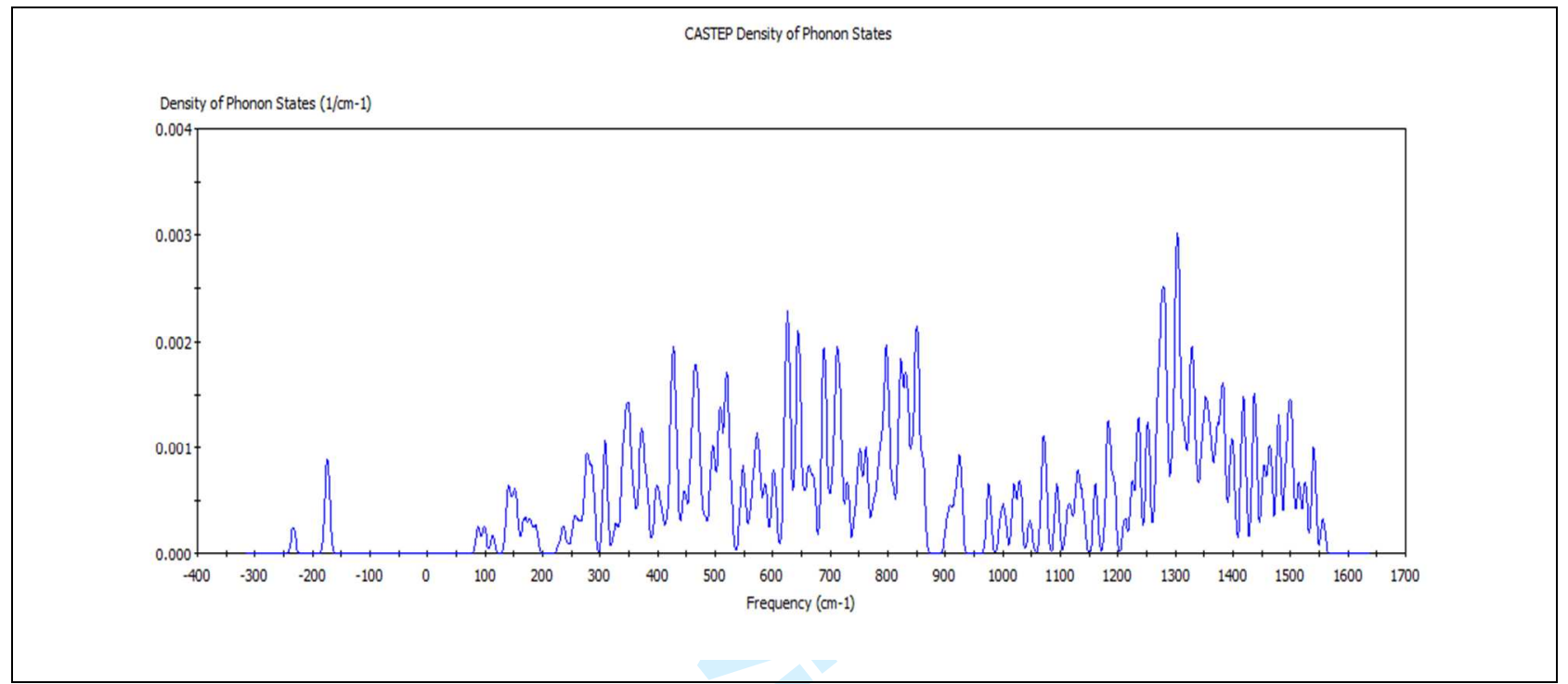

Fig.2 a) Phonon Density of States

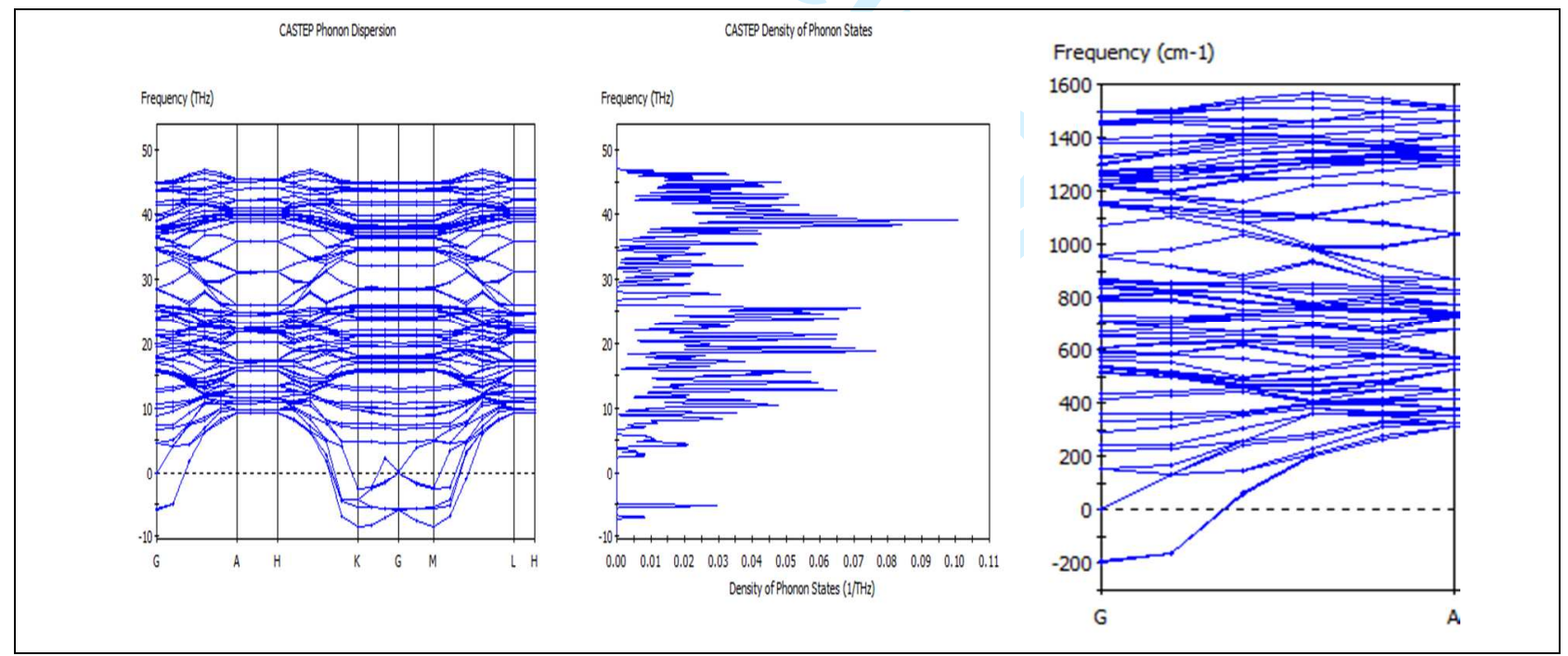

Fig.2 b) Phonon Dispersion Curves

It can be seen that SWCNT phonon density of states has a number of sharp peaks (van Hove singularities) due to its 1-D structure. The low frequency phonon modes are more reflective of the symmetry of carbon nanotubes, while higher frequency modes are more reflective of carbon-carbon bonding. 
The soft modes around $-200 \mathrm{~cm}^{-1}$ are somehow surprising as the structure is duly relaxed. Appearance of these soft modes at negative frequencies is due to ignorance of strong on-site interactions of localized electrons in SWCNTs which is not correctly described by GGA approximation. The on-site Coulomb interactions are particularly strong for localized $d$ and f electrons, but can be also important for $p$ localized orbitals. The strength of the on-site interactions is usually described by parameters $\mathrm{U}$ (on site Coulomb) and $\mathrm{J}$ (on site exchange). The soft modes present at $U=0$ will disappear at $U>0$ as force constants are dependent on the $U$ value. Also, SWCNTs possess a long interaction range and missing force constants to the far away atoms also give rise to fake soft modes. However, the simulated structure has been perfectly optimized and these negative frequencies are rather small and will not generate large enough forces to destroy the dynamical stability of the system and hence can be overlooked.

\subsection{Vibrational Modes}

Wrapping a graphene sheet into a SWCNT results into a periodic boundary condition along the circumference which quantizes the corresponding wave vector. As a result, the 2-D graphene phonon modes are further split into a large number of sub-bands. If the SWCNT contains N atoms per unit cell, there are $3 \mathrm{~N}$ phonon modes in total, many of which are degenerated [26]. As the simulated SWCNT consisted of 36 atoms, total 108 phonon modes were obtained. Out of these 108 phonon modes 6 are redundant. There are four acoustic modes: a longitudinal acoustic, two degenerate transverse acoustic and a twist mode. In addition, there are various radial, bending, stretching, twisting and helical modes in the 98 modes in the optical branch (Fig.3). Some of the vibrational modes of an $(8,0)$ SWCNT have been shown in fig. 4 .However, these can be generalized to depict vibrations in any zigzag SWCNT.

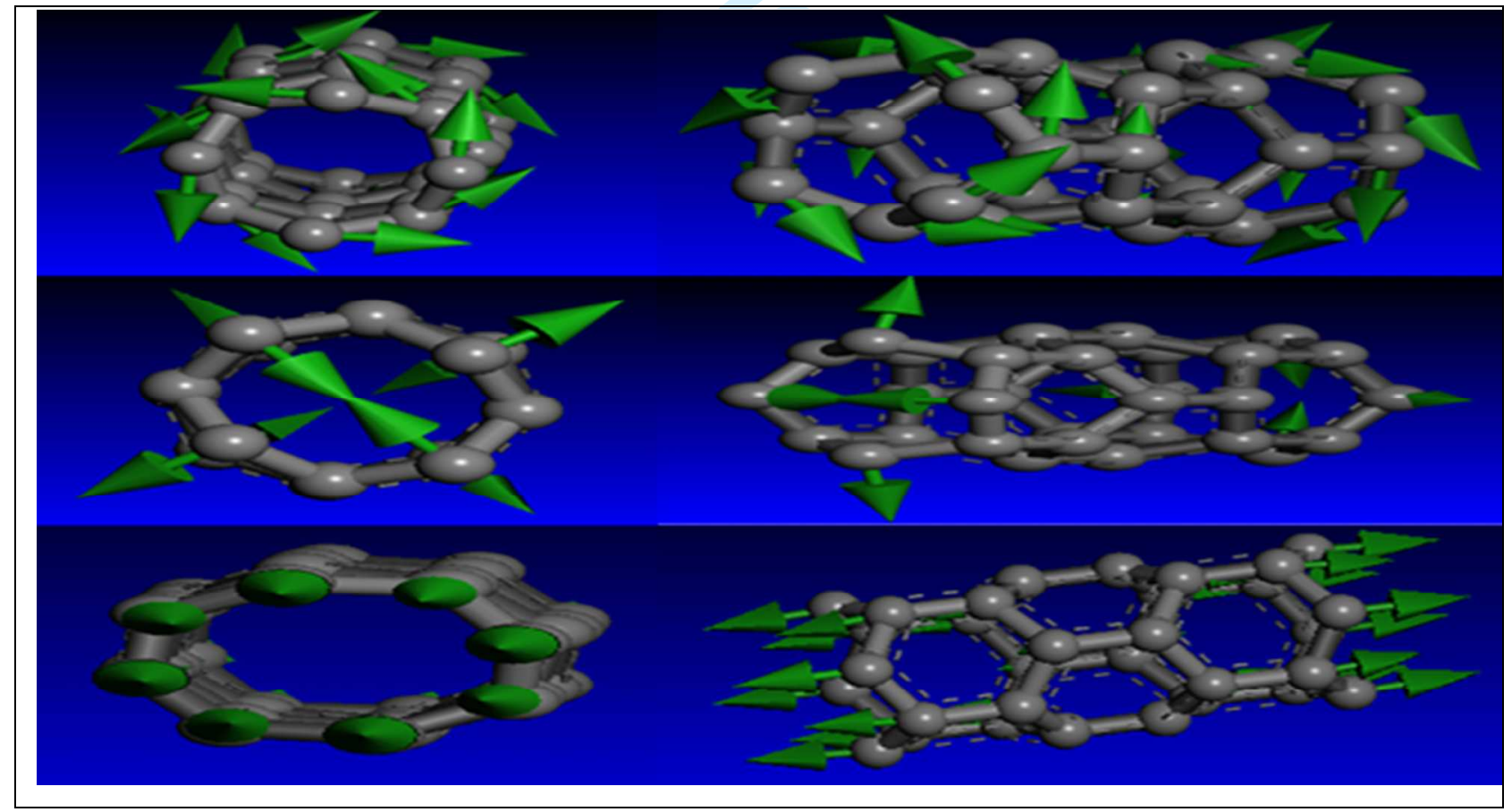

Fig.3 A glimpse of some of the vibrational modes

These SWCNT phonon modes can be viewed as a mixture of 2-D graphene phonon modes [31]. There are four acoustic modes in-stead of three; a longitudinal acoustic (LA) mode, two degenerate transverse acoustic (TA) modes and a twist mode [32]. The radial breathing mode (RBM) is another important low-frequency mode, corresponding 
to radial expansion-contraction of the nanotube. It is similar to the out-of plane TA mode in graphene with zero energy at the center of the Brillouin zone, but becomes a mode with a finite energy due to the curvature.

\subsection{Group Theory Analysis}

Group theory analysis of eigenvectors reveals that the simulated structure displays Point Group 27: D6h, $6 / \mathrm{mmm}, 6 / \mathrm{m} \mathrm{2} / \mathrm{m} \mathrm{2} / \mathrm{m}$ symmetries and allows scope for 24 symmetry operations. Frequency calculations at 23 wavevectors (q-points) were performed using Gonze variational method with TPA preconditioning scheme. A number of symmetry operations are apparent for $(9,0)$ SWCNT: We can rotate the tube by $360^{\circ} / 9=40^{\circ}$ about its $z$-axis, the nanotube axis. There is a series of so-called primed rotations, which are perpendicular to $z$. The most obvious one has its rotational axis normal to the paper. We can also reflect the lattice at a mirror plane normal to $z$ going through the centers of the hexagons (horizontal mirror plane). And, finally, one can identify a set of vertical mirror planes including the nanotube axis and the carbon-carbon bonds parallel to it. These symmetry operations do not require a translation of the tube by a fraction of its lattice vector. There is, however, a second set of operations in a $(9,0)$ nanotube that preserve the lattice. They involve translations, i.e., screw axes and glide planes. We can also apply a rotation followed by a translation. This generates the helix twisting around the circumference of the tube. In addition, there are two sets of $C_{2}$ rotations perpendicular to $z$, one horizontal $\left(\sigma_{\mathrm{h}}\right)$ and two sets of vertical $\left(\sigma_{\mathrm{v}}, \sigma_{\mathrm{v}}{ }^{\prime}\right)$ mirror planes.

The symmetry of single walled carbon nanotubes is described by the so-called line groups. Line groups are the full space groups of one-dimensional systems including translations in addition to the point-group symmetries like rotations or reflections. Every nanotube with particular $(n, m)$ chirality belongs to a different line group [33-36]. To obtain the phonon symmetry, it is sufficient to analyze the points of the K set only. The phonon symmetry at other points in the BZ can be obtained from the compatibility relations. Character table obtained from group theory analysis of eigenvectors summarizes these symmetry considerations (Table-2).

(TABLE-2)

\section{CHARACTER TABLE}

\begin{tabular}{|c|c|c|c|c|c|c|c|c|c|c|c|c|c|}
\hline & Mullikan & $\mathbf{E}$ & $\mathbf{2 C}_{\mathbf{3}}$ & $\mathbf{C}_{\mathbf{2}}$ & $\mathbf{2 C}_{\mathbf{6}}$ & $\mathbf{3 C}_{\mathbf{2}}$ & $\mathbf{3 C}_{\mathbf{2}}{ }^{\prime}$ & $\mathbf{I}$ & $\mathbf{2 S}_{\mathbf{3}}$ & $\boldsymbol{\sigma}_{\mathbf{h}}$ & $\mathbf{2 S}_{\mathbf{6}}$ & $\boldsymbol{\sigma}_{\mathbf{v}}$ & $\boldsymbol{\sigma}_{\mathbf{v}}{ }^{\prime}$ \\
\hline $\mathrm{E}_{1} \mathrm{~g}$ & 9 & 2 & -1 & -2 & 1 & 0 & 0 & 2 & -1 & -2 & 1 & 0 & 0 \\
\hline $\mathrm{A}_{2} \mathrm{~g}$ & 4 & 1 & 1 & 1 & 1 & -1 & -1 & 1 & 1 & 1 & 1 & -1 & -1 \\
\hline $\mathrm{E}_{1} \mathrm{u}$ & 9 & 2 & -1 & -2 & 1 & 0 & 0 & -2 & 1 & 2 & -1 & 0 & 0 \\
\hline $\mathrm{A}_{2} \mathrm{u}$ & 5 & 1 & 1 & 1 & 1 & -1 & -1 & -1 & -1 & -1 & -1 & 1 & 1 \\
\hline $\mathrm{E}_{2} \mathrm{~g}$ & 9 & 2 & -1 & 2 & -1 & 0 & 0 & 2 & -1 & 2 & -1 & 0 & 0 \\
\hline $\mathrm{B}_{2} \mathrm{u}$ & 5 & 1 & 1 & -1 & -1 & 1 & -1 & -1 & -1 & 1 & 1 & -1 & 1 \\
\hline $\mathrm{B}_{1} \mathrm{u}$ & 4 & 1 & 1 & -1 & -1 & -1 & 1 & -1 & -1 & 1 & 1 & 1 & -1 \\
\hline $\mathrm{E}_{2} \mathrm{u}$ & 9 & 2 & -1 & 2 & -1 & 0 & 0 & -2 & 1 & -2 & 1 & 0 & 0 \\
\hline $\mathrm{A}_{1} \mathrm{~g}$ & 5 & 1 & 1 & 1 & 1 & 1 & 1 & 1 & 1 & 1 & 1 & 1 & 1 \\
\hline $\mathrm{B}_{2} \mathrm{~g}$ & 4 & 1 & 1 & -1 & -1 & 1 & -1 & 1 & 1 & -1 & -1 & 1 & -1 \\
\hline $\mathrm{B}_{1} \mathrm{~g}$ & 5 & 1 & 1 & -1 & -1 & -1 & 1 & 1 & 1 & -1 & -1 & -1 & 1 \\
\hline $\mathrm{A}_{1} \mathrm{u}$ & 4 & 1 & 1 & 1 & 1 & 1 & 1 & -1 & -1 & -1 & -1 & -1 & -1 \\
\hline
\end{tabular}


Out of the 12 vibrational symmetry modes listed in the character table (Table-2), some are Raman active, some are IR active while some are neither Raman active nor IR active.

\subsection{Raman Active Modes}

While in chiral tubes there are six Raman active high energy modes $\left(2 \mathrm{~A}_{1}, 2 \mathrm{E}_{1}, 2 \mathrm{E}_{2}\right)$, in achiral tubes only three of the high energy modes are Raman active i.e. $\mathrm{E}_{1} \mathrm{~g}, \mathrm{E}_{2} \mathrm{~g}$ and $\mathrm{A}_{1} \mathrm{~g}$. From polarization dependent Raman experiments on both unoriented and aligned single-wall tubes, it is known that the phonon modes which contribute most to the Raman signal have $\mathrm{A}_{1} \mathrm{~g}$ symmetry. The bands corresponding to these symmetries obtained from ab initio phonon calculations are tabulated in 'Table-3'.

(TABLE-3)

MODES CORRESPONDING TO RAMAN ACTIVE VIBRATIONAL SYMMETRIES

\begin{tabular}{|c|c|c|c|c|c|}
\hline \multicolumn{2}{|c|}{$\mathbf{E}_{\mathbf{1}} \mathbf{g}$} & \multicolumn{2}{|c|}{$\mathbf{E}_{\mathbf{2}} \mathbf{g}$} & \multicolumn{2}{c|}{$\mathbf{A}_{\mathbf{1}} \mathbf{g}$} \\
\hline Frequency $\left(\mathrm{cm}^{-1}\right)$ & Degeneracy & Frequency $\left(\mathrm{cm}^{-1}\right)$ & Degeneracy & Frequency $\left(\mathrm{cm}^{-1}\right)$ & Degeneracy \\
\hline 530.14 & 2 & 151.39 & 2 & 357.46 & 1 \\
\hline 648.08 & 2 & 332.78 & 2 & 520.39 & 1 \\
\hline 850.35 & 2 & 605.79 & 2 & 1150.36 & 1 \\
\hline 857.73 & 2 & 653.56 & 2 & 1222.97 & 1 \\
\hline 869.11 & 2 & 956.96 & 2 & - & - \\
\hline 1162.35 & 2 & 1258.96 & 2 & - & - \\
\hline 1266.78 & 2 & 1274.62 & 2 & - & - \\
\hline 1499.29 & 2 & 1299.44 & 2 & - & - \\
\hline- & - & 1395.22 & 2 & - & - \\
\hline
\end{tabular}

Raman spectra were also obtained through simulation using the GGA (RPBE) exchange correlation and norm conserving pseudo-potential (Fig. 4).

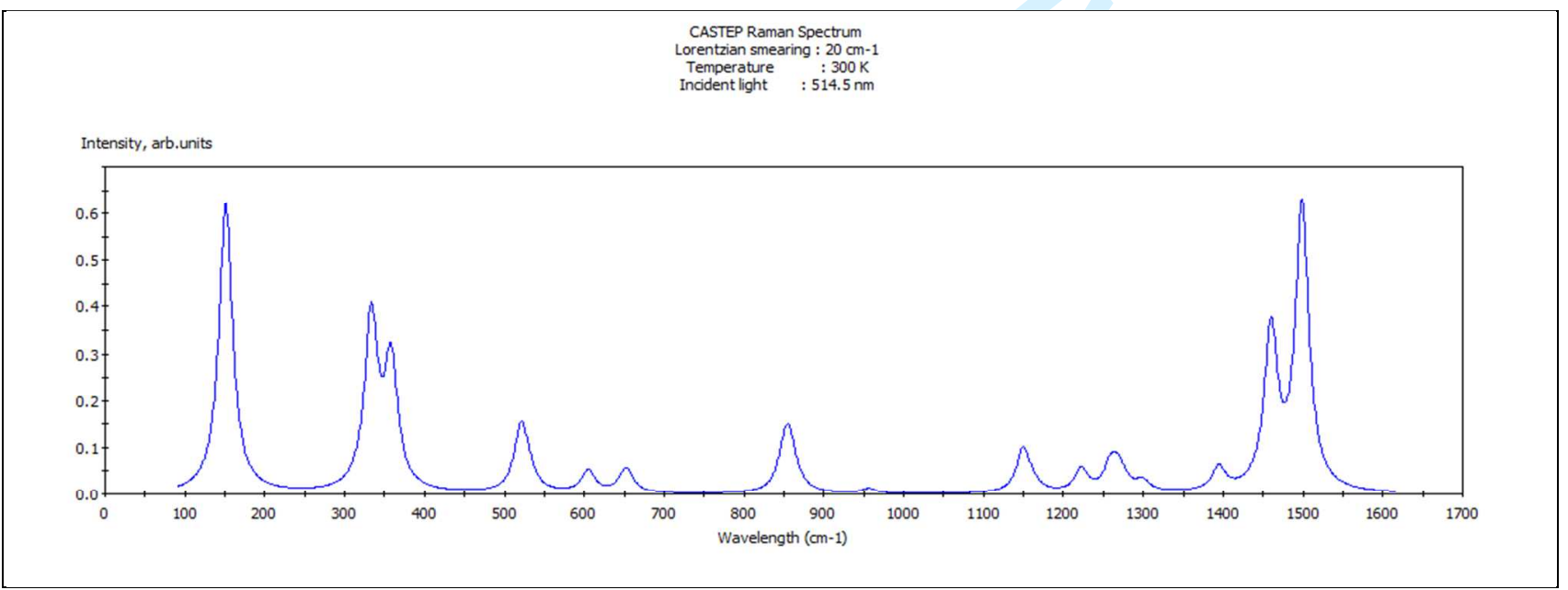

Fig.4 Raman Spectrum of $(9,0)$ zigzag SWCNT 
The Raman spectrum obtained (Fig. 4) reveals peaks corresponding to radial breathing mode (RBM) in the low frequency region (100-300 $\left.\mathrm{cm}^{-1}\right)$ and the peak corresponding to typical graphitic tangential stretch (G-band) in the high frequency region (around $1590 \mathrm{~cm}^{-1}$ ). Some peaks are also obtained in the frequency range $500-1300 \mathrm{~cm}^{-1}$ which are normally not visible in the experimental reports. However, Chen et al. and Y.Wang et al. have reported these peaks in their experimental findings as well $[37,38]$.

\subsection{IR Active Modes}

Normal vibrational modes having $\mathrm{E}_{1 \mathrm{u}}$ and $\mathrm{A}_{2 \mathrm{u}}$ symmetry are found to be IR active. The bands corresponding to these symmetries as obtained through ab initio calculation are listed in 'Table-4' (redundant band excluded):

(TABLE-4)

MODES CORRESPONDING TO IR ACTIVE VIBRATIONAL SYMMETRIES

\begin{tabular}{|c|c|c|c|}
\hline \multicolumn{2}{|c|}{$\mathbf{E}_{\mathbf{1}} \mathbf{u}$} & \multicolumn{2}{|c|}{$\mathbf{A}_{\mathbf{2}} \mathbf{u}$} \\
\hline Frequency $\left(\mathrm{cm}^{-1}\right)$ & Degeneracy & Frequency $\left(\mathrm{cm}^{-1}\right)$ & Degeneracy \\
\hline 418.48 & 2 & 0.48 & 1 \\
\hline 436.34 & 2 & 563.93 & 1 \\
\hline 605.31 & 2 & 861.02 & 1 \\
\hline 1072.75 & 2 & 950.10 & 1 \\
\hline 1212.85 & 2 & 1147.71 & - \\
\hline 1239.66 & 2 & - & - \\
\hline 1266.11 & 2 & - & - \\
\hline 1449.56 & 2 & - & \\
\hline
\end{tabular}

The calculated intensities of IR emission at various frequencies of IR active vibrational modes when plotted against the concerned mode frequency led to the IR spectrum (Fig. 5).

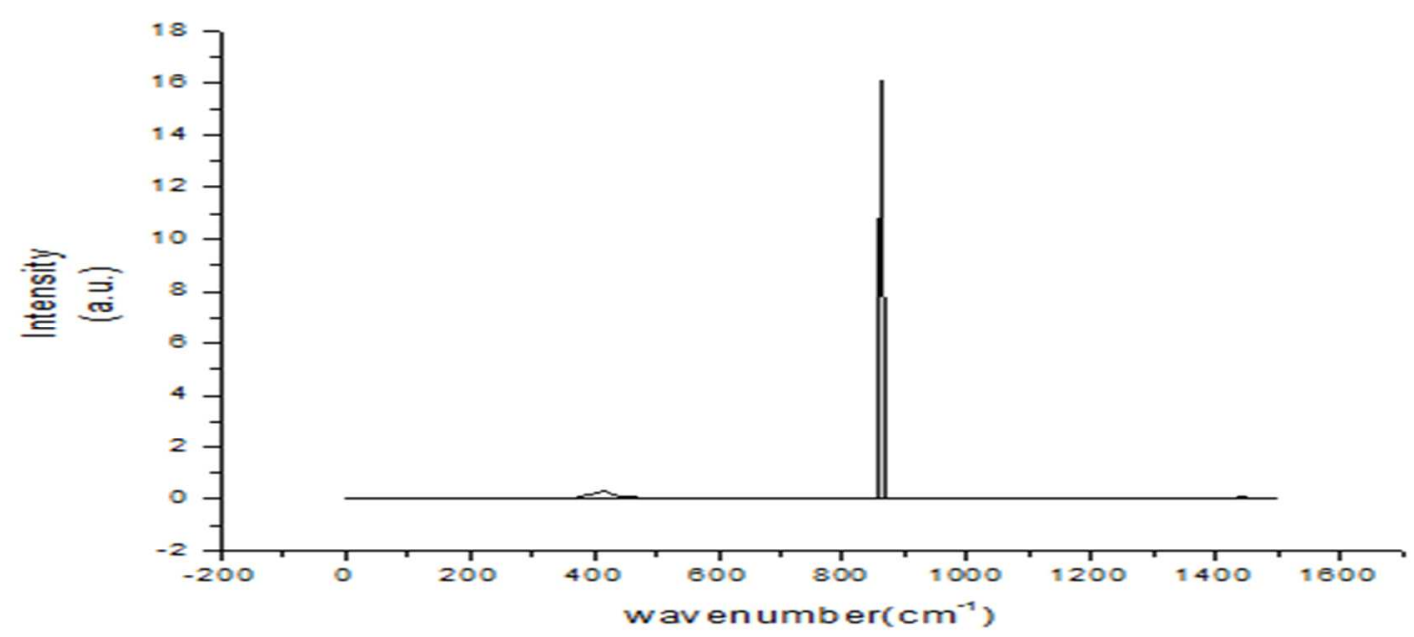

Fig. 5 IR Spectrum of $(9,0)$ zigzag SWCNT

Spectrum (Fig.5) shows a sharp IR peak around $850 \mathrm{~cm}^{-1}$ as is expected of all the substances with graphitic bonds as per the study made by U.Kuhlmann et. al. [39]. 


\section{Conclusion}

Phonon dispersion curves and phonon densities of states (PDOS) of a zigzag SWCNT were calculated over a full range of wave-vectors using ab initio quantum mechanical numerical method. Symmetries associated with the 108 phonon modes obtained were analyzed and the symmetry modes that are IR active or Raman active were identified. Such kind of spectroscopic study may prove to be an effective tool for quick and reliable analytical characterization of SWCNTs and provide a deeper insight into the fundamental phenomena responsible for various electronic, optical and thermodynamic properties associated with these 1-D tubules. It may further allow the study of lattice dynamical contribution to a broader range of structural and thermodynamic phenomena than is feasible with IR and Raman spectroscopy.

\section{References}

1. X Zhang et al. Carbon 94903 (2015)

2. J Bauschlicher, W Charles, A Ricca Physical Review B 70115409 (2004)

3. A Ricca and C W Bauschlicher Chemical physics 324455 (2006)

4. E Cakmak, X Fang,O Yildiz, P D Bradford, T K Ghosh Carbon 89113 (2015)

5. S J Sung, T Kim, S J Yang, J Y Oh, C R Park, New insights into the oxidation of single-walled carbon nanotubes for the fabrication of transparent conductive films Carbon 81525 (2015)

6. X Xu, J Zhai, Y Chen, H Zhu, H., L Li, S Ruan \& Z Tang Carbon 9584 (2015)

7. M Irita \& Y Homma 461282 (2014)

8. L X Benedict, V H Crespi, S G Louie \& M L Cohen Physical Review B 5214935 (1995)

9. H Ishii et al. Nature $426 \mathbf{5 4 0}$ (2003)

10. D Connétable, G M Rignanese, J C Charlier, \& X Physical review letters 94015503 (2005)

11. Rols, S. et al. Physical review letters 855222 (2000)

12. C Thomsen Physical Review B 614542 (2000)

13. J Maultzsch, S Reich \& C Thomsen Physical Review B 64121407 (2001)

14. J Maultzsch, S Reich \& C Thomsen Physical Review B 65233402 (2002)

15. V Zolyomi \& J Kürti Physical Review B 66073418 (2002)

16. G G Samsonidze et al. Physical review letters 90027403 (2003)

17. M Oron-Carl, F Hennrich, M M Kappes,H V Löhneysen, \& R Krupke Nano letters 5 1761(2005)

18. C L Kane et al. EPL (Europhysics Letters) 41683 (1998)

19. Y Zhen, C L Kane \& C Dekker Physical Review Letters 842941 (2000)

20. S Hidekatsu \& T Ando Physical review B 65235412 (2002)

21. A Sédéki, L G Caron \& C Bourbonnais Physical Review B 626975 (2000)

22. M Sugano,A Kasuya, K Tohji, Y Saito \& Y Nishina Chemical physics letters 292 575(1998)

23. Q Ding, Q Jiang, Z Jin, D Tian FULLERENE SCIENCE \& TECHNOLOGY 431 (1996)

24. A Charlier, E McRae, M F Charlier, A Spire \& S Forster Physical Review B 576689 (1998)

25. R A Jishi, L Venkataraman, M S Dresselhaus \& G Dresselhaus Chemical Physics Letters 20977 (1993)

26. D Sánchez-Portal, E Artacho, J M Soler, A Rubio \& P Ordejón Physical Review B 5912678 (1999)

27. O Dubay \& G Kresse Physical Review B 67035401 (2003)

28. J Marquina, C Power, J González \& J M Broto Advances in Materials Physics and Chemistry 3178 (2013)

29. Y Matsuda, J Tahir-Kheli \& W A Goddard III The Journal of Physical Chemistry Letters 12946 (2010)

30. V Y Davydov et al. Physical Review B 5812899 (1998)

31. M S Dresselhaus \& P C Eklund Advances in Physics 49705 (2000)

32. J Hone Carbon Nanotubes Springer Berlin Heidelberg 273 (2001)

33. I Božović, N Božović \& M. Damnjanović Physical Review B 626971 (2000)

34. O E Alon Physical Review B 63201403 (2001)

35. C Thomsen \& S Reich Light Scattering in Solid IX Springer Berlin Heidelberg 115 (2006)

36. V Zólyomi, J Koltai, J Kürti, H Kuzmany, V Basiuk \& S Irle DFT Calculations on Fullerenes and Carbon Nanotubes (India: Research Signpost) ed. A V Basiuk \& S Irle (2008) 
37. G Chen et al. Journal of nanoscience and nanotechnology 2621 (2002)

38. Y Wang, B Zhang, Q Jin, B Li, D Ding, \& X Cao Spectrochimica Acta Part A: Molecular and Biomolecular Spectroscopy 681149 (2007)

39. U Kuhlmann, H Jantoljak, N Pfänder, P Bernier, C Journet \& C Thomsen Chemical Physics Letters 294 237 (1998) 